\title{
Assembly of Atomically Precise Silver Nanoclusters into Nanocluster-Based Frameworks
}

\author{
Mohammad J. Alhilaly, ${ }^{\dagger,+\perp}$ Ren-Wu Huang, ${ }^{\dagger, \ddagger}$ Rounak Naphade, ${ }^{\dagger, \ddagger}$ Badriah Alamer, ${ }^{\dagger, \dagger}$
}

Mohamed Nejib Hedhili, ${ }^{\S}$ Abdul-Hamid Emwas, ${ }^{\S}$ Partha Maity, ${ }^{\dagger, \ddagger(0)}$ Jun Yin, ${ }^{\dagger \odot}$

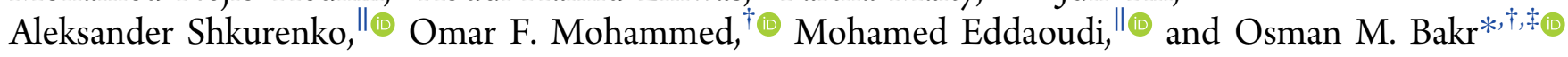

${ }^{\dagger}$ Division of Physical Sciences and Engineering, ${ }^{\ddagger}$ KAUST Catalysis Center (KCC), ${ }^{\S}$ Core Laboratories, and "Functional Materials Design, Discovery and Development Research Group (FMD3), Advanced Membranes and Porous Materials Center, King Abdullah University of Science and Technology (KAUST), Thuwal 23955-6900, Saudi Arabia

${ }^{\perp}$ Department of Physics, College of Sciences, Imam Mohammad Ibn Saud Islamic University (IMSIU), Riyadh, 11623, Saudi Arabia

\section{Supporting Information}

ABSTRACT: Here, we demonstrate an approach to synthesizing and structurally characterizing three atomically precise anion-templated silver thiolate nanoclusters, two of which form one- and two-dimensional structural frameworks composed of bipyridine-linked nanocluster nodes (referred to as nanocluster-based frameworks, NCFs). We describe the critical role of the chloride $\left(\mathrm{Cl}^{-}\right)$template in controlling the nanocluster's nuclearity with atomic precision and the effect of a single $\mathrm{Ag}$ atom difference in the nanocluster's size in controlling the NCF dimensionality, modulating the optical properties, and improving the thermal stability. With atomically precise assembly and size control, nanoclusters could be widely adopted as building blocks for the construction of tunable cluster-based framework materials.

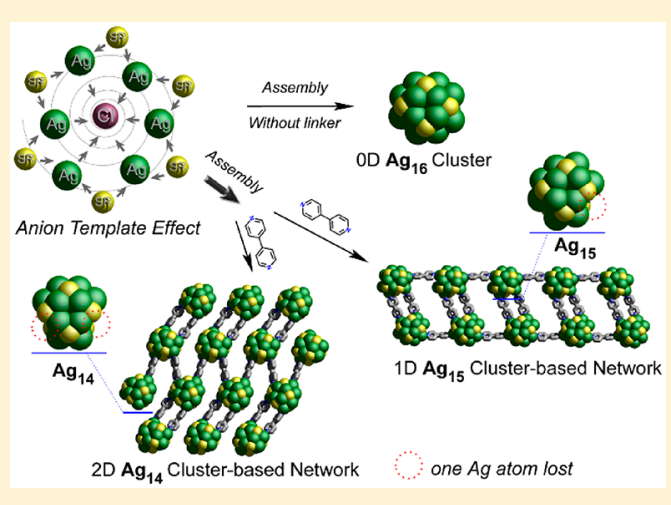

\section{INTRODUCTION}

One primary aim of nanotechnology is the bottom-up programmed assembly of materials from nanosized building blocks with molecular precision and control. Unfortunately, most nanoparticles are atomically inhomogeneous, precluding their use as building blocks for such assembly. Atomically precise nanoparticles, also known as nanoclusters (NCs), are thus highly prized in the nanochemist's tool kit because they make nanoparticles amenable to supramolecular assembly, property control, and materials interrogation (e.g., periodic structures amenable to single-crystal structure determination). Researchers have made tremendous strides in synthesizing metal and metal chalcogenide NCs with unique structures and distinct physicochemical properties; ${ }^{1-5}$ for example, an impressive level of control over the size and surface ligand chemistry of such NCs has been achieved. However, relatively little progress has been made in developing and understanding the supramolecular chemistry associated with the assembly of NCs into crystalline solids. In fact, NCs reported to date typically crystallize into relatively trivial close-packed structures that lack diversity. The ability to control the NCs' coordination number affords access to distinct secondary building units (SBUs) with assorted geometries and connectivity, defined by the arrangement of points of extensions on the NCs and the prospective to construct periodic extended solids with finetuned porosity, reactivity, and conductivity as well as optical and mechanical properties. $^{6-12}$
Silver NCs stabilized with ligands (such as thiolate, alkynyl, or phosphine $)^{13-27}$ are ideal candidates for developing chemical approaches to the controlled self-assembly of solids. The size, composition, and surface chemistry of such NCs are reasonably well understood; however, there are enormous benefits to be gained from controlling the self-assembly modes of these NCs, stemming from their prospective applications in catalysis, ${ }^{28-30}$ fluorescence sensing, ${ }^{31}$ and bioimaging ${ }^{32}$ as well as antimicrobials. ${ }^{33}$ The assembly of NCs into atomically precise NC-based frameworks would combine advantages from both NCs and metal-organic frameworks (MOFs), including providing high-nuclearity cluster nodes, high structural tunability, highly connected frameworks with more structural complexity, large channels, and more robustness. These potential advantages are attractive for a wide range of potential applications including sensing, catalysis, and gas separation. ${ }^{6,34}$ Recently, $\mathrm{Ag}$ thiolate NCs, such as $\mathrm{Ag}_{10}, \mathrm{Ag}_{12}$, and $\mathrm{Ag}_{14},{ }^{34-36}$ have been shown to assemble into one-, two-, and threedimensional frameworks in which the NCs form nodes connected by pyridyl-type organic linkers. In addition, several assembled structures of anion-templated $\mathrm{Ag}$ thiolate nanoclusters networked by short inorganic anions have also been reported. $^{37,38}$ The solvents used have also been found to play a critical role in the assemblies' morphology. ${ }^{39}$ Despite advances

Received: March 6, 2019

Published: May 28, 2019 
in the synthesis of Ag-cluster-based MOFs in terms of boosting stability and enhancing optical properties, it remains challenging to produce atomically precise cluster-based frameworks (also referred to here as nanocluster-based frameworks, NCFs) with different dimensionalities. Indeed, studies on controlling cluster size in assembled networks with atomic precision or on the significant roles played by size and anion templates, ${ }^{40}$ which are believed to affect the dimensionality and resulting properties, have yet to be reported.

In this work, we present a strategy for extending the size and dimensional control over $\mathrm{Ag}(\mathrm{I})$ thiolate NCs. In particular, we report three new crystal structures: a single Ag nanocluster (0D NC) and two Ag nanocluster-based frameworks (1-D NCF and 2-D NCF). Our approach relies on $\mathrm{Cl}^{-}$as a template for NC growth in conjunction with a suitable organic linker, 4,4' bipyridine (bpy), in the case of 1-D NCF and 2-D NCF. More specifically, when no organic linker (no bpy) is used, a $\mathrm{Ag}_{16}$ cluster is attained, which crystallizes as a discrete zerodimensional structure (0-D NC). When bpy is added, depending on the ratio of the ligands (mainly the bpy linker) to the $\mathrm{Ag}$ thiolate complex, we found that two distinct clusters form, $\mathrm{Ag}_{15}$ and $\mathrm{Ag}_{14}$, each adopting an MOF-like structure with specific dimensionality (i.e., 1-D NCF and 2-D NCF, respectively). The three cluster units in 0-D NC, 1-D NCF, and 2-D NCF share notable similarities. In particular, the units possess a similar geometrical structure in which the skeletons of cluster cores can be considered to be complete or incomplete square gyrobicupolas (Figure 1) and the protecting
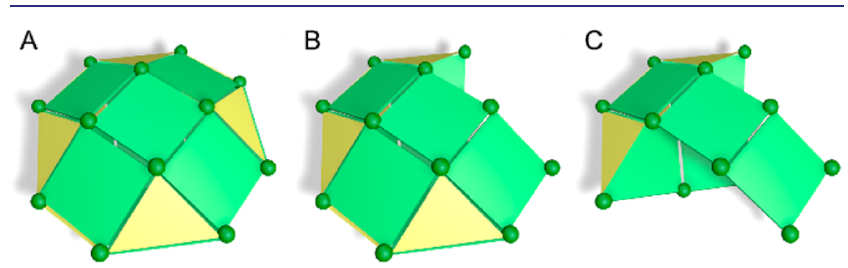

Figure 1. Geometrical structures of the cores of (A) 0-D NC, (B) 1-D NCF, and (C) 2-D NCF.

ligand shell of each cluster core contains a combination of the thiolate $\left({ }^{t} \mathrm{BuS}^{-}\right)$and trifluoroacetate $\left(\mathrm{CF}_{3} \mathrm{COO}^{-}\right)$ligands as well as coordinated solvent molecules. As the ratio of the ligands to the $\mathrm{Ag}$ thiolate complex increases (in the presence of bpy linker), clusters begin to distort more to accommodate structures of higher connectivity (and thus confined spacing). These distortions lead to a sequential loss of a single surface Ag atom as the NC adopts one- and two-dimensional assembled networks of $\mathrm{Ag}_{15}$ and $\mathrm{Ag}_{14}$ (1-D NCF and 2-D NCF), respectively. All three structures (one silver NC and two cluster-based assembled networks) exhibit qualitatively distinct characteristics in photoluminescence (PL) and thermal stability. Specifically, higher-dimensional structures show enhanced PL and higher thermal stability.

Our approach represents a new method for controlling the nuclearity and dimensionality of self-assembled NCs, leading to novel atomically precise cluster-based MOF-like assembled materials and at the same time modulating their optical properties. With improved control and understanding of the structures' self-assembly and size, we anticipate that metal NCs will provide myriad modular building blocks, with assorted shapes and connectivities, for the practice of reticular chemistry.

\section{RESULTS AND DISCUSSION}

Synthesis and Characterization of 0-D NC, 1-D NCF, and 2-D NCF. As illustrated in Scheme 1, a zero-dimensional

Scheme 1. Synthesis of Ag NC- and NC-Based Frameworks
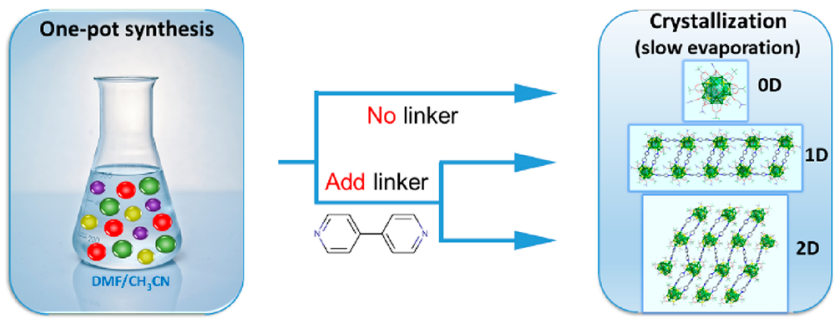

Ag NC (0-D NC) and two Ag NC-based frameworks (1-D NCF and 2-D NCF) were synthesized by a chemical reaction of the precursors using a one-pot approach. The chemical reaction initially occurred between the premade $\left[\mathrm{Ag}-\mathrm{S}^{t} \mathrm{Bu}\right]_{n}$ complex with silver trifluoroacetate in $\mathrm{CH}_{3} \mathrm{CN}$ solvent to produce a homogeneous mixture under stirring. Then, a soluble dimethylformamide (DMF) solution of tetraphenylphosphonium chloride $\left(\mathrm{PPh}_{4} \mathrm{Cl}\right)$ was added to form the $\mathrm{Ag}$ NCs. It is noted that the DMF solvent plays a critical role in facilitating the templating effect of $\mathrm{Cl}^{-}$. To facilitate the assembly of the $\mathrm{Ag}$ clusters into one- and two-dimensional networks (1-D NCF and 2-D NCF, respectively), bpy was added to the reaction mixture and utilized as an organic linker. By applying specific ratios of the precursors (see the Experimental Section for the synthesis details), rodlike greenemitting crystals of 2-D NCF were obtained in high yield. By increasing the ratio of the independent ligands (bpy and $\mathrm{AgCF}_{3} \mathrm{COO}$ precursors) to the $\mathrm{Ag}$ thiolate complex, we found that the crystallization system tended to grow predominantly large 1-D NCF crystals and small 2-D NCF crystals (as a coproduct). After the crystallization of the resulting clear solutions of the filtrates by slow evaporation at room temperature, high-quality single crystals suitable for singlecrystal X-ray diffraction (SCXRD) were obtained after 7-12 days. The crystal structures of 0-D NC, 1-D NCF, and 2-D NCF were studied by SCXRD, and the phase purities were evaluated by PXRD. Furthermore, the optical properties and thermal stability of the structures were studied. The synthesis and crystallization details of all three products can be found in the Experimental Section.

Single-Crystal Structures of 0-D NC, 1-D NCF, and 2-D NCF. $\left[\mathrm{Ag}_{16} \mathrm{Cl}\left(\mathrm{S}^{t} \mathrm{Bu}\right)_{8}\left(\mathrm{CF}_{3} \mathrm{COO}\right)_{7}(\mathrm{DMF})_{4}\left(\mathrm{H}_{2} \mathrm{O}\right)\right] \cdot 1.5(\mathrm{DMF}) \quad(0-\mathrm{D}$ NC). First, a crystal of 0-D NC was evaluated by SCXRD. An analysis of the crystallographic data showed that 0-D NC crystallizes in monoclinic space group $\mathrm{C} 2 / c$ (Table S1). The structure of 0-D NC is essentially a silver cluster with a $\mathrm{Ag}_{16}$ skeleton welded by argentophilic interactions; the $\mathrm{Ag}_{16}$ cluster is templated by one enclosed $\mathrm{Cl}^{-}$in the center and forms a distorted square gyrobicupola (29th Johnson solid, J29), ${ }^{41}$ which is a polyhedron with 8 triangular and 10 distorted square faces (Figure 1A). Among these polygons, each square face is capped by a ${ }^{t} \mathrm{BuS}^{-}$ligand (except for the top and bottom faces) with a consistent $\mu_{4}-\eta^{1}: \eta^{1}: \eta^{1}: \eta^{1}$ ligation mode to link four silver ions with $\mathrm{Ag}-\mathrm{S}$ distances in the range of 2.368(2)-2.598(2) $\AA$ (Figures 2, top). The skeleton is also protected by seven $\mathrm{CF}_{3} \mathrm{COO}^{-}$auxiliary ligands (Figure $\mathrm{S} 1$ ). Five of those ligands bind to the equatorial plane (octagonal $\mathrm{Ag}_{8}$ ) of the polyhedron 

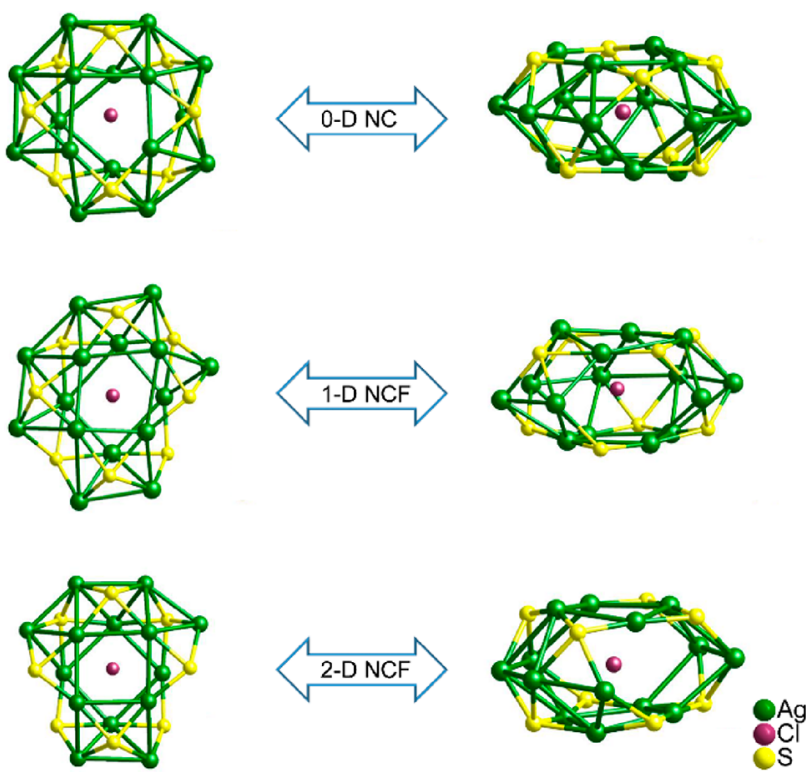

Figure 2. Top view vs side view comparison between core structures of (top) 0-D NC, (middle) 1-D NCF, and (bottom) 2-D NCF.

through Ag-O bonds adopting the $\mu_{2}-\eta^{1}: \eta^{1}$ mode (Ag-O: 2.19(3)-2.474(6) $\AA$ ). The remaining two $\mathrm{CF}_{3} \mathrm{COO}^{-}$ligands cap the upper $\left(\mu_{3}-\eta^{1}: \eta^{2}\right.$ mode) and lower $\left(\mu_{2}-\eta^{1}: \eta^{1}\right.$ mode) square faces with $\mathrm{Ag}-\mathrm{O}$ bonds in the ranges of $2.40(1)-$ $2.68(2)$ and 2.483(8)-2.69(3) $\AA$, respectively. The protecting ligand shell also contains four DMF molecules and one $\mathrm{H}_{2} \mathrm{O}$ molecule coordinated in the equatorial plane (Figure S4, left). In addition, the $\mathrm{Ag}_{16}$ cluster structure includes one and a half molecules of the cocrystallized DMF solvents (Figure S5, left). Interestingly, within the skeleton, the distances between the central $\mathrm{Cl}^{-}$and peripheral Ag atoms, in the range of 2.789(2)3.608(2) $\AA$, are much longer than those of conventional Ag$\mathrm{Cl}$ bonds (approximately 2.6 $\AA$ ). A similar situation has been observed for alkynyl-protected silver clusters. ${ }^{14,42,43}$ The anion templating effect of $\mathrm{Cl}^{-}$is quite important for the assembly of the skeleton structure. It is worth noting that the argentophilic interactions also play a crucial role in the stabilization of the silver cluster skeleton. The observed Ag ‥Ag distances range from $2.919(2)$ to $3.286(2) \AA$, which are shorter than twice the van der Waals radius of silver (3.44 $\AA$ ), indicating significant argentophilic interactions.

$\left[\mathrm{Ag}_{15} \mathrm{Cl}\left(\mathrm{S}^{t} \mathrm{Bu}\right)_{8}\left(\mathrm{CF}_{3} \mathrm{COO}\right)_{5.67}\left(\mathrm{NO}_{3}\right)_{0.33}(\mathrm{bpy})_{2}(\mathrm{DMF})_{2}\right] \cdot 4.3-$ (DMF). $\mathrm{H}_{2} \mathrm{O}$ (1-D NCF). Crystallographic data analysis of 1-D NCF showed that its crystal structure contains a $\mathrm{Ag}_{15}$ cluster also templated by $\mathrm{Cl}^{-}$and it belongs to triclinic space group $\bar{P} 1$ (Table S2). The skeleton of the $\mathrm{Ag}_{15}$ cluster core is composed of an incomplete distorted square gyrobicupola through the argentophilic interactions, with $\mathrm{Ag} \cdots \mathrm{Ag}$ bond distances in the range of 2.912(1)-3.381(1) $\AA$ as a result of one missing $\mathrm{Ag}$ atom; the polyhedron contains six triangular and eight square faces (Figure 1B). The skeleton is protected by eight ${ }^{t} \mathrm{BuS}^{-}$ ligands that adopt two types of coordination modes-six adopt the $\mu_{4}-\eta^{1}: \eta^{1}: \eta^{1}: \eta^{1}$ ligation mode and two adopt the $\mu_{3}-\eta^{1}: \eta^{1}: \eta^{1}$ ligation mode-where the $\mathrm{Ag}-\mathrm{S}$ distances are in the range of $2.338(3)-2.576(2) \AA$. The structure also features a clear distortion, as shown in Figure 2 (middle). In contrast to the four coordinated DMF molecules in the $\mathrm{Ag}_{16} \mathrm{NC}$ (0-D NC), only two coordinated DMF molecules were observed in the $\mathrm{Ag}_{15}$ cluster of 1-D NCF. Likewise, in the previous structure the $\mathrm{Ag}_{15}$ is coprotected by $\mathrm{CF}_{3} \mathrm{COO}^{-}$auxiliary ligands: four of them are coordinated in the equatorial plane in the $\mu_{2}-\eta^{1}: \eta^{1}$ mode with $\mathrm{Ag}-\mathrm{O}$ distances in the range of $2.299(6)-3.054(8)$ $\AA$; the ligands are found in the upper $\left(\mu_{3}-\eta^{1}: \eta^{2}\right.$ mode $)$ and lower axial positions $\left(\mu_{2}-\eta^{1}: \eta^{1}\right.$ mode) with $\mathrm{Ag}-\mathrm{O}$ distances in the ranges of $2.450(8)-2.709(7)$ and $2.399(8)-2.60(2) \AA$, respectively. The number of $\mathrm{CF}_{3} \mathrm{COO}^{-}$auxiliary ligands was also observed to decrease proportionally as one $\mathrm{Ag}$ atom was lost to balance the charge. Interestingly, this compound is found to contain the substitutional disorder of one auxiliary ligand bonded to the equatorial $\mathrm{Ag}$ atom, with an occupancy of 0.67 for $\mathrm{CF}_{3} \mathrm{COO}^{-}$and 0.33 for $\mathrm{NO}_{3}^{-}$. The unanticipated $\mathrm{NO}_{3}{ }^{-}$may be derived from the residual silver nitrate in the $\left[\mathrm{Ag}-\mathrm{S}^{t} \mathrm{Bu}\right]_{n}$ complex precursor (Figure S4, middle). Finally, each $\mathrm{Ag}_{15}$ cluster is connected to three adjacent such clusters through four bpy linkers to form an infinite one-dimensional (1D) ladder-like structure. In this structure, dual-cluster units, each consisting of two clusters and two juxtaposed bpy monomer pillars, are further combined by single-bpy bridges along the $b$ axis only. The dual-cluster unit is inclined relative
A

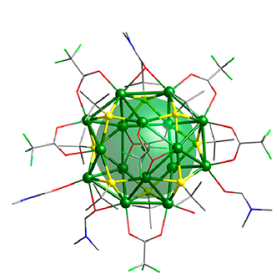

C

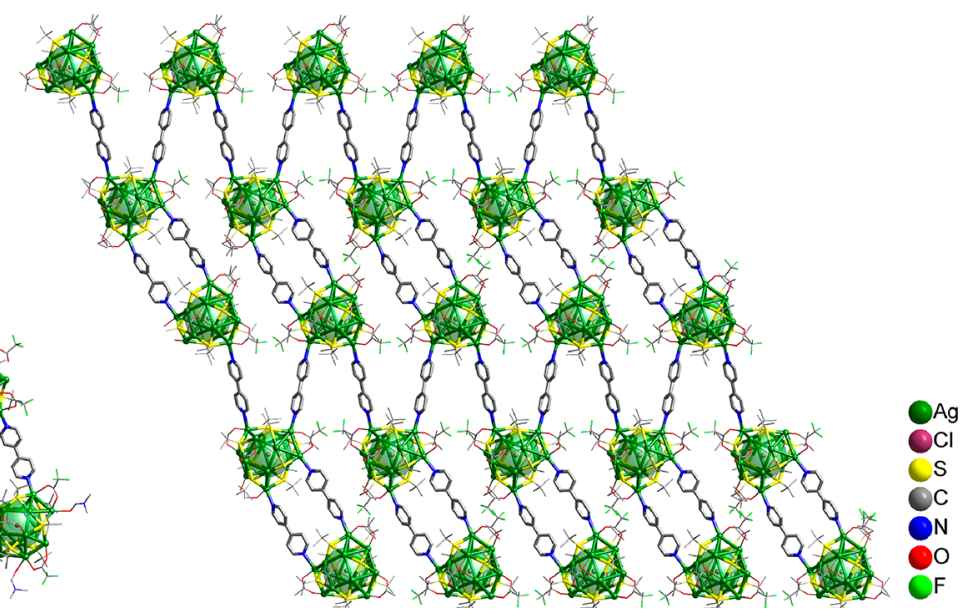

Figure 3. Crystal structures of (A) 0-D NC, (B) 1-D NCF, and (C) 2-D NCF. Free (cocrystallized) DMF molecules are not shown. The green semitransparent spheres in the silver clusters are shown purely as a guide. Hydrogens have been omitted for clarity. 

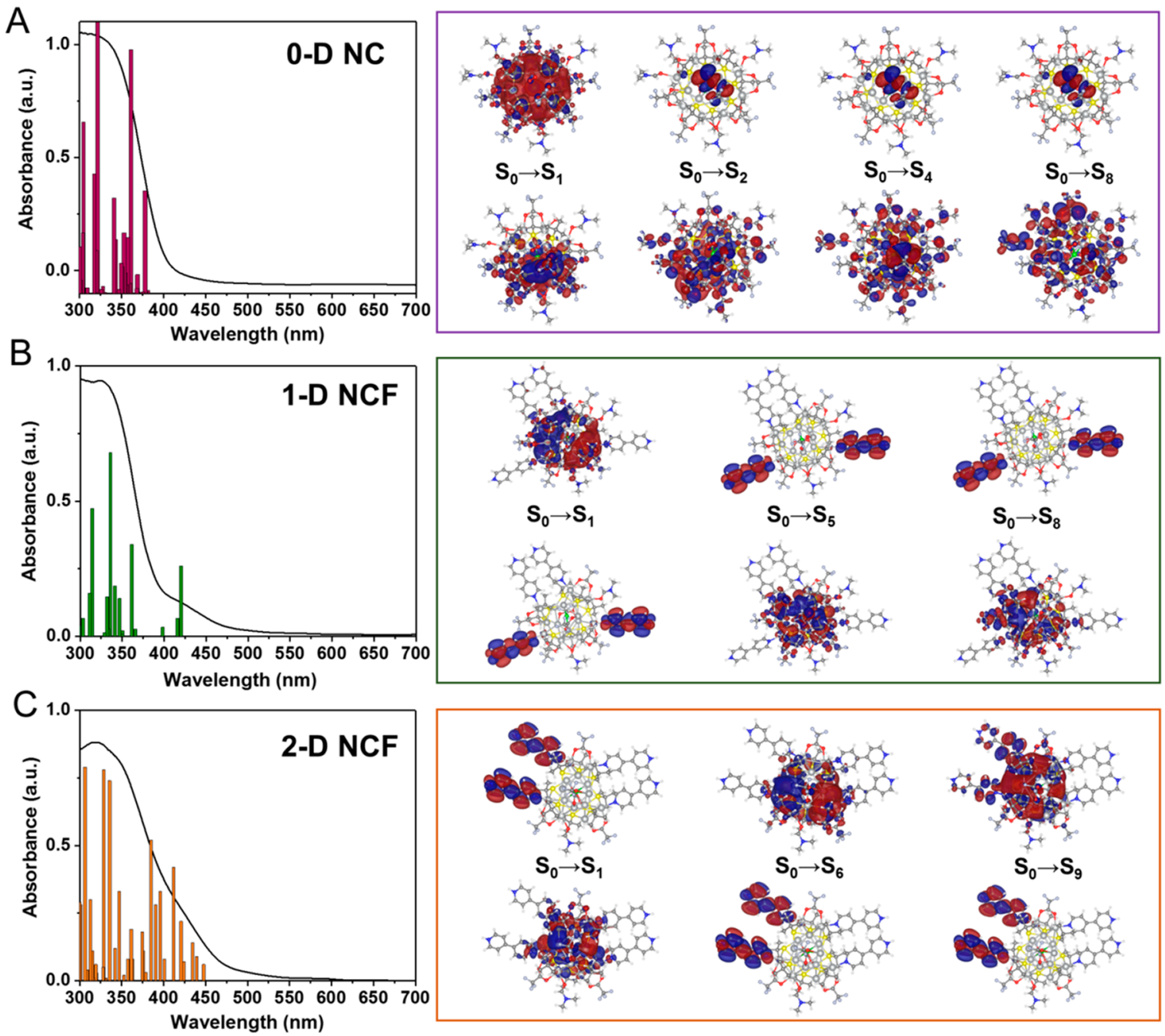

Figure 4. Experimental absorption spectra (solid black lines) with calculated optical transition wavelengths and oscillator strengths (vertical lines) using the TDDFT method, the dominant natural transition orbital (NTO) hole, and particle pairs (isovalue $=0.02 \mathrm{e} \cdot \AA^{-3}$ ) for the main singlet excited states of (A) 0-D NC, (B) 1-D NCF, and (C) 2-D NCF.

to the single-bpy bridges at an acute angle of approximately $70^{\circ}$ (Figure 3B).

$\left[A g_{14} \mathrm{Cl}\left(\mathrm{S}^{t} \mathrm{Bu}\right)_{8}\left(\mathrm{CF}_{3} \mathrm{COO}\right)_{5}(\mathrm{bpy})_{2}(\mathrm{DMF})\right] \cdot 2(\mathrm{DMF})$ (2-D NCF). Crystallizing in triclinic space group $\bar{P} 1$ (Table S3), 2-D NCF is formed by a $\mathrm{Ag}_{14}$ cluster that, similarly to 0-D NC and 1-D NCF, is also assembled by a templating chloride. The $\mathrm{Ag}_{14}$ skeleton is considered to be an incomplete square gyrobicupola and assembles into a two-dimensional structure of networked $\mathrm{Ag}_{14}$ cluster nodes (Figures 2, bottom and Figure 3C). Because 2 of the original $16 \mathrm{Ag}$ atoms are missing, the $\mathrm{Ag}_{14}$ skeleton features only 4 triangular and 6 square faces constructed via sparse argentophilic interactions ( $\mathrm{Ag} \cdots \mathrm{Ag}$ distances in the range of $2.890(1)-3.4072(8) \AA$ ) and is coprotected by 8 ${ }^{t} \mathrm{BuS}^{-}$ligands, $5 \mathrm{CF}_{3} \mathrm{COO}^{-}$auxiliary ligands, and only 1 coordinated DMF molecule (Figures S4 and S5, right). We found that the eight ${ }^{t} \mathrm{BuS}^{-}$ligands adopt two types of coordination modes: four adopt the $\mu_{4}-\eta^{1}: \eta^{1}: \eta^{1}: \eta^{1}$ ligation mode, and the other four adopt the $\mu_{3}-\eta^{1}: \eta^{1}: \eta^{1}$ ligation mode. The range of $\mathrm{Ag}-\mathrm{S}$ distances is 2.34(2)-2.558(2) $\AA$. The five $\mathrm{CF}_{3} \mathrm{COO}^{-}$ligands can also be classified into two groups. Similar to the structures described above, the equatorial ligand and one axial $\mathrm{CF}_{3} \mathrm{COO}^{-}$ligand are coordinated in the $\mu_{2}-\eta^{1}: \eta^{1}$ mode, while the second axial ligand is coordinated in the $\mu_{3}$ - $\eta^{1}: \eta^{2}$ ligation mode. The Ag-O distances range from 2.288(6) to 2.93(1) A. Furthermore, each $\mathrm{Ag}_{14}$ cluster coordinates to four bpy linkers. Consequently, two clusters and two parallel bpy linkers are employed to construct a dual-cluster unit similar to that in 1-D NCF. Each dual-cluster unit is further connected to four others in the upper and lower rows by single-bpy bridges to form a $2 \mathrm{D}$ framework in which the $\mathrm{Ag}_{14}$ clusters are regarded as the nodes of the 2D framework (Figure $3 \mathrm{C})$. In other words, two of the $\mathrm{Ag}_{14}$ clusters are directed toward the same neighbor, forming a dual-cluster unit, as observed in 1-D NCF. From a topological point of view, the two linkers there act as one edge (Figure S7B). Because the next two bpy spacers are directed toward different $\mathrm{Ag}_{14}$ clusters, two-dimensional framework formation is observed in which each $\mathrm{Ag}_{14}$ cluster is a 3-c node and the entire twodimensional framework is a 3-c, 2-periodic edge transitive net called hcb (Figure S7C). Finally, the adjacent 2D layers are further extended into a $3 \mathrm{D}$ supramolecular structure via weak hydrogen bonds between the terminal $t$-butyl and trifluoromethyl groups on the surface of the adjacent layers (Figure S8). The layers are slightly offset with respect to each other, thus two types of channels in the 2-D NCF crystal structure are observed: the channels oriented along the $\left[\begin{array}{ll}1-1 & 0\end{array}\right]$ 
direction are parallel to the layers, whereas the channels oriented along the crystallographic $a$ axis penetrate them. Two noncoordinated DMF molecules were localized in the channels, and the contribution of the significantly delocalized electron density was excluded from the refinement by the PLATON SQUEEZE ${ }^{44}$ procedure.

Optical Absorption and Photoluminescence Properties of 0-D NC, 1-D NCF, and 2-D NCF. We recorded the optical absorption spectra of 0-D NC in solution and in the solid state (Figure S9). Crystals of 0-D NC display a clear absorption band in the UV region ( 200-350 nm), as shown in Figure S9 (top). Crystals of 1-D NCF and 2-D NCF also exhibit absorption bands in the UV region, but both show an additional shoulder peak at $\sim 420 \mathrm{~nm}$ (Figures S10 and S11). This shoulder is observed only for 1-D NCF and 2-D NCF possibly because of residual solvent (DMF molecules) within the networked cluster nodes, as the shoulder peak becomes less intense (or nearly disappears) with more efficient drying of the crystals.

Crystals of 2-D NCF exhibit clear green emission at $\sim 530$ $\mathrm{nm}$ with excitation at $365 \mathrm{~nm}$ at room temperature ( 298 K) under ambient conditions (Figure S12). In contrast, the emissions of 0-D NC and 1-D NCF at room temperature are not visually detectable. Indeed, we could not observe any steady-state PL under UV light $(365 \mathrm{~nm})$. Nevertheless, we were able to detect weak emission of 0-D NC and 1-D NCF at lower temperatures using temperature-dependent photoluminescence (TDPL). The maximum emission wavelength of the TDPL of 0 -D NC occurs at $\sim 535 \mathrm{~nm}$, with no notable spectral shift with varying temperature; the spectrum also shows a shoulder peak at $\sim 430 \mathrm{~nm}$ (Figure S13). We observed a clear, systematic decrease in the 0-D NC emission intensity with increasing temperature. 1-D NCF crystals display emission spectra similar to those of 0-D NC crystals, exhibiting the same emission at $\sim 535 \mathrm{~nm}$, with no clear shift with changing temperature, and the shoulder peak $(\sim 430 \mathrm{~nm})$ is also observed (Figure S14). However, 0-D NC shows sensitivity to UV light; specifically, a change in color is observed as a result of degradation induced by UV light (at an excitation wavelength of $365 \mathrm{~nm}$ ). We also examined the TDPL of 2-D NCF crystals, which exhibit distinctly stronger emission, as shown in Figure S15. A systematic spectral red shift was observed with an increase in temperature from $77 \mathrm{~K}$ to room temperature, with the emission wavelength varying from $\sim 511$ to $540 \mathrm{~nm}$ under excitation at $325 \mathrm{~nm}$. Furthermore, given the appreciable PL signal of 2-D NCF crystals, a PL lifetime of $0.32 \mu$ s was measurable (excitation at $372 \mathrm{~nm}$ ), which may originate from the radiative recombination of the excited triplet state (Figure S16). ${ }^{34,35}$ The results of the optical investigation of the three species clearly show that 2-D NCF, which possesses the highest dimensionality among the three structures, displays enhanced PL properties. Such PL enhancement could be attributed to the linker-to-cluster chargetransfer state as explained from DFT calculations (vide infra).

DFT Calculations. We performed time-dependent density functional theory (TDDFT) calculations to study the major optical transitions of 0-D NC, 1-D NCF, and 2-D NCF. Overall, as shown in Figure 4, our calculated optical transition wavelengths and oscillator strengths are reasonably good matches with the experimental absorption spectra. For 0-D NC, most absorption peaks with a large oscillator strength $\left(S_{1}\right.$, $S_{2}, S_{4}$, and $S_{8}$ ) can be assigned to the electronic transitions of a "hole" delocalized in the $\mathrm{Ag}_{16}$ cluster to the "particle" localized partially in the cluster core using natural transition orbital (NTO) analysis. However, for both 1-D NCF and 2-D NCF, the excitation induced by light irradiation can effectively shift the electronic distribution from the hole/particle localized in organic linkers to the delocalized particle/hole in the cluster core. In contrast to the cluster-to-linker feature of the $S_{1} \rightarrow S_{0}$ transition in 1-D NCF, the enhanced PL intensity observed for 2-D NCF could be attributed to linker-to-cluster chargetransfer excitation.

Other Characterizations. PXRD measurements confirmed the crystallinity and phase purity of the three structures based on the agreement between the experimental and simulated spectra (Figure S17). Thermogravimetric analysis (TGA) measurements were conducted to monitor the thermal stability of the structures. As shown in Figure S18, the main weight loss for 0-D NC occurs at $\sim 124{ }^{\circ} \mathrm{C}$. This loss corresponds to the complete decomposition of the building blocks of $\mathrm{Ag}_{16}$ clusters. The TGA of 1-D NCF showed a large drop corresponding to the main loss of mass at $\sim 150{ }^{\circ} \mathrm{C}$. We found that less distinct weight loss occurs at low temperatures for better air-dried samples; therefore, the initial loss is due to residual DMF solvent. The main loss most likely corresponds to the complete decomposition of the Ag cluster-building units. In 2-D NCF, similar TGA behavior was observed, but the main loss occurred at $\sim 160{ }^{\circ} \mathrm{C}$, which indicates that the structure is more robust. Thus, the TGA results indicate a clear improvement in thermal stability and robustness with increasing dimensionality from 0-D NC to 2-D NCF. Additionally, we evaluated the thermal stability of NCFs through monitoring the PL retention and recovery (from 0 to $100{ }^{\circ} \mathrm{C}$ ). The data show that 2-D NCF has retained most of its $\mathrm{PL}$, indicating that 2-D NCF is more thermally stable than 1-D NCF (Figure S19).

Fourier transform infrared (FTIR) spectroscopy was performed on powder samples. The broad peak at around $3400 \mathrm{~cm}^{-1}$ in the 0-D NC spectrum is assigned to the $\mathrm{OH}$ stretching vibration of a water molecule (which confirms the coordinated $\mathrm{H}_{2} \mathrm{O}$ in 0-D NC only). However, the peak between 2800 and $3000 \mathrm{~cm}^{-1}$ is observed in all structures-0D NC, 1-D NCF, and 2-D NCF-and corresponds to the terminal $\mathrm{C}-\mathrm{H}$ of the tert-butyl functional group in the thiolate ligand. The obtained sharp peak at $\sim 1640 \mathrm{~cm}^{-1}$ is observed in all three structures and is assigned to the $\mathrm{C}=\mathrm{O}$ stretching vibration of the DMF molecules in the crystal structures. In addition, an extra shoulder peak at $\sim 1595 \mathrm{~cm}^{-1}$ is observed only in 1-D NCF and 2-D NCF, which can be assigned to the $\mathrm{C}-\mathrm{N}$ stretching vibration from the bpy linker (Figure S20).

Furthermore, X-ray photoelectron spectroscopy (XPS) was performed to characterize the chemical composition of the surface of a 2-D NCF crystal sample and to determine the oxidation state of chlorine. The survey spectrum of the 2-D NCF crystal sample shows that $\mathrm{Ag}, \mathrm{S}, \mathrm{F}, \mathrm{Cl}, \mathrm{O}$, and $\mathrm{C}$ were detected (Figure S21). The high-resolution XPS spectrum of the $\mathrm{Cl} 2 \mathrm{p}$ core level was obtained from the 2-D NCF crystal sample. The $\mathrm{Cl} 2 \mathrm{p}$ region shows one doublet positioned at 198.2 and $199.8 \mathrm{eV}$ corresponding to the $\mathrm{Cl} 2 \mathrm{p}_{3 / 2}$ and $\mathrm{Cl} 2 \mathrm{p}_{1 / 2}$ spin-orbit split components, respectively (Figure S22, top). For comparison, the high-resolution XPS spectrum of the $\mathrm{Cl}$ $2 \mathrm{p}$ core level was obtained from the $\mathrm{PPh}_{4} \mathrm{Cl}$ precursor. The $\mathrm{Cl}$ $2 \mathrm{p}$ region shows one doublet situated at 196.2 and $197.8 \mathrm{eV}$ corresponding to the $\mathrm{Cl} 2 \mathrm{p}_{3 / 2}$ and $\mathrm{Cl} 2 \mathrm{p}_{1 / 2}$ spin-orbit split components, respectively (Figure S22, bottom). These binding energies correspond to the ionic form of free chlorine $\left(\mathrm{Cl}^{-}\right)$in 
$\mathrm{PPh}_{4} \mathrm{Cl}^{45}$ The shift in the $\mathrm{Cl} 2 \mathrm{p}_{3 / 2}$ component from low binding energy at $196.2 \mathrm{eV}$ for the $\mathrm{PPh}_{4} \mathrm{Cl}$ precursor to high binding energy at $198.2 \mathrm{eV}$ for the 2-D NCF crystal sample indicates that $\mathrm{Cl}^{-}$interacts strongly with the silver skeleton around it in the 2-D NCF crystal compared to the free $\mathrm{Cl}^{-}$ ions in $\mathrm{PPh}_{4} \mathrm{Cl}$.

Consistent with the SCXRD results, solid-state ${ }^{13} \mathrm{C}$ nuclear magnetic resonance (NMR) spectroscopy was also performed for the crystals of 0-D NC, 1-D NCF, and 2-D NCF. Different DMF- $\mathrm{CH}_{3}$ peaks were observed at 31.16 and $32.22 \mathrm{ppm}$ and at 45.99 and $47.91 \mathrm{ppm}$, which were associated with the bondedDMF and free-DMF environments, respectively. Moreover, the carbonyl C peak was observed to split into two peaks at 161.74 ppm due to free DMF molecules and formed one extra peak at 165.34 due to coordinated DMF. Because of F-C coupling, the $\mathrm{CF}_{3}$ peak split into different lines at $114.68,116.59,118.71$, and $122.25 \mathrm{ppm}$, while a peak attributed to COOAg was observed at $159.82 \mathrm{ppm}$. The strong peak observed at 36.88 ppm was assigned to methyl groups of ${ }^{t} \mathrm{BuS}^{-}$, and a quaternary carbon peak appeared at $51.09 \mathrm{ppm}$. A comparison of the 0-D NC spectrum with the 1-D NCF and 2-D NCF spectra shows extra peaks in the aromatic region (122.31, 146.26, and 151.56 $\mathrm{ppm})$, which can be assigned to the aromatic peaks of the bpy linker (Figure S23). ${ }^{46}$

In addition, to further corroborate the molecular formula and purity of $\mathbf{0 - D} \mathbf{N C}$, electrospray ionization mass spectrometry (ESI-MS) measurements were performed on the crystals of $\mathbf{0}-\mathrm{D}$ NC dissolved in $\mathrm{CH}_{3} \mathrm{CN}$ solvent (as shown in Figure S24). The assignments of the mass peaks demonstrate a clear match between isotopic patterns of the experimental and simulated mass spectra (Figure S24a-c). The deduced molecular formula from ESI-MS is in good agreement with the crystal structure obtained by SCXRD.

\section{CONCLUSION}

We report three novel structures of atomically precise chloridetemplated $\mathrm{Ag}(\mathrm{I})$ thiolate nanoclusters and nanocluster-based frameworks (0-D NC, 1-D NCF, and 2-D NCF). By employing four main reaction levers $\left(\mathrm{Cl}^{-}\right.$anion template, DMF solvent, bpy linker, and reactant/precursor ratio), we successfully achieved the controlled assembly of a well-defined zero-dimensional $\mathrm{Ag}_{16}$ cluster (0-D NC) and two cluster-based assembled networks, $\mathrm{Ag}_{15}$ (1-D NCF) and $\mathrm{Ag}_{14}$ (2-D NCF) with atomic-level control over cluster size and dimensionality. The change in dimensionality offers a distinct way to tune the optical and PL properties (as well as to improve the thermal stability) of cluster-based frameworks. This study paves the way for synthesizing new functional nanomaterials of clusterbased MOFs with full atomic precision and utilizing their tunable properties for a wide range of potential applications.

\section{EXPERIMENTAL SECTION}

Materials. All the following chemicals and solvents were purchased and used without further purification: silver nitrate $\left(\mathrm{AgNO}_{3}, 99 \%\right.$, Aldrich), silver trifluoroacetate $\left(\mathrm{AgCF}_{3} \mathrm{COO}, 98 \%\right.$, Aldrich), tetraphenylphosphonium chloride $\left(\mathrm{PPh}_{4} \mathrm{Cl}, 98 \%\right.$, Aldrich), tert-butyl mercaptan (99\%, Aldrich), trimethylamine (99\%, pure, ACROS), 4,4'-bipyridine (bpy, 98\%, Aldrich), dichloromethane (DCM, HPLC grade, VWR), methanol ( $\mathrm{MeOH}, \mathrm{HPLC}$ grade, VWR), N,N-dimethylformamide (DMF, HPLC grade, Aldrich), and acetonitrile $\left(\mathrm{CH}_{3} \mathrm{CN}\right.$, HPLC grade, Aldrich).

Synthesis of the $\left[\mathrm{Ag}-\mathrm{S}^{t} \mathrm{Bu}\right]_{n}$ Complex. The complex was prepared according to a previously reported method ${ }^{47}$ but with some modifications. First, $\mathrm{AgNO}_{3}(3 \mathrm{mmol})$ was completely dissolved in 20
$\mathrm{mL}$ of $\mathrm{MeOH}$ by sonication. In another vial, tert-butyl mercaptan ( 3 $\mathrm{mmol}$ ) was dissolved in $10 \mathrm{~mL}$ of DCM and directly added to the methanolic solution of $\mathrm{AgNO}_{3}$. Then, triethylamine $(\sim 1 \mathrm{mmol})$ was added quickly to the reaction mixture under stirring. The reaction was continued at room temperature (RT) in the dark overnight. The next day, the sample was centrifuged at $8000 \mathrm{rpm}$ for $5 \mathrm{~min}$; the white precipitate of the complex was washed at least five times with $\mathrm{MeOH}$ and then stored under vacuum for further use.

Synthesis and Crystallization of 0-D NC. In a glass vial, $\sim 0.3$ mmol of $\left[\mathrm{Ag}-\mathrm{S}^{t} \mathrm{Bu}\right]_{n}$ and $\sim 0.2 \mathrm{mmol}$ of $\mathrm{AgCF}_{3} \mathrm{COO}$ were mixed in 5 $\mathrm{mL}$ of $\mathrm{CH}_{3} \mathrm{CN}$ and stirred for $\sim 5 \mathrm{~min}$. Then, $4 \mathrm{~mL}$ of a soluble DMF solution of $\mathrm{PPh}_{4} \mathrm{Cl}(\sim 0.04 \mathrm{mmol})$ was immediately added to the reaction mixture with stirring, and the reaction was continued for $\sim 2$ $\mathrm{h}$ at RT. After filtration, the filtrate was allowed to evaporate slowly at room temperature in the dark under ambient conditions. After 9-12 days, colorless crystals were obtained. The average yield of 0-D NC was $47.8 \%$ (based on $\mathrm{Ag}$ ).

Synthesis and Crystallization of a 1-D NCF and 2-D NCF Mixture. In a glass vial, $\sim 0.34 \mathrm{mmol}$ of $\left[\mathrm{Ag}-\mathrm{S}^{t} \mathrm{Bu}\right]_{n}, 0.26 \mathrm{mmol}$ of $\mathrm{AgCF}_{3} \mathrm{COO}$, and $\sim 0.38 \mathrm{mmol}$ of bpy were mixed together in $5 \mathrm{~mL}$ of $\mathrm{CH}_{3} \mathrm{CN}$ with stirring for $\sim 5 \mathrm{~min}$. Then, $4 \mathrm{~mL}$ of a soluble DMF solution of $\mathrm{PPh}_{4} \mathrm{Cl}(\sim 0.06 \mathrm{mmol})$ was added to the reaction mixture, and the reaction was continued under stirring for $\sim 2 \mathrm{~h}$ at room temperature. The filtrate was then allowed to evaporate slowly at RT in the dark under ambient conditions. Large reddish crystals of 1-D NCF in addition to small 2-D NCF crystals were obtained after 1 week of crystallization. The average yield of 1-D NCF was 35.6\% (based on Ag).

Synthesis and Crystallization of 2-D NCF. In a glass vial, 0.3 $\mathrm{mmol}$ of $\left[\mathrm{Ag}-\mathrm{S}^{t} \mathrm{Bu}\right]_{n}, 0.2 \mathrm{mmol}$ of $\mathrm{AgCF}_{3} \mathrm{COO}$, and $0.3 \mathrm{mmol}$ of bpy were mixed together in $5 \mathrm{~mL}$ of $\mathrm{CH}_{3} \mathrm{CN}$ with stirring for $\sim 5 \mathrm{~min}$. Then, $4 \mathrm{~mL}$ of a soluble DMF solution of $\mathrm{PPh}_{4} \mathrm{Cl}(0.04 \mathrm{mmol})$ was added to the reaction mixture, and the reaction was continued under stirring for $\sim 2 \mathrm{~h}$ at room temperature. The filtrate was then allowed to evaporate slowly at RT in the dark under ambient conditions. Colorless rodlike crystals were obtained after 1 week of crystallization. The average yield of 2-D NCF was 55.6\% (based on Ag).

\section{ASSOCIATED CONTENT}

\section{Supporting Information}

The Supporting Information is available free of charge on the ACS Publications website at DOI: 10.1021/jacs.9b02486.

$$
\begin{aligned}
& \text { Ag16_0D (CIF) } \\
& \text { Ag15_1D (CIF) } \\
& \text { Ag14_2D (CIF) }
\end{aligned}
$$

Instrumentation, UV-vis, steady-state PL, PL lifetime, PXRD, XPS, TGA, FTIR, ${ }^{13} \mathrm{C}$ NMR, ESI-MS, and analyses of crystal structures of 0-D NC, 1-D NCF, and 2-D NCF (PDF)

\section{AUTHOR INFORMATION}

\section{Corresponding Author}

*osman.bakr@kaust.edu.sa

ORCID

Rounak Naphade: 0000-0002-3504-8552

Partha Maity: 0000-0002-0293-7118

Jun Yin: 0000-0002-1749-1120

Aleksander Shkurenko: 0000-0001-7136-2277

Omar F. Mohammed: 0000-0001-8500-1130

Mohamed Eddaoudi: 0000-0003-1916-9837

Osman M. Bakr: 0000-0002-3428-1002

\section{Notes}

The authors declare no competing financial interest. 


\section{ACKNOWLEDGMENTS}

The authors sincerely thank the following people for their help and dedication at various stages of this project: Ayalew Assen, Karim Adil, and Osama Shekhah. The financial support for this work was provided by KAUST.

\section{REFERENCES}

(1) Jin, R.; Zeng, C.; Zhou, M.; Chen, Y. Atomically precise colloidal metal nanoclusters and nanoparticles: fundamentals and opportunities. Chem. Rev. 2016, 116 (18), 10346-10413.

(2) Chakraborty, I.; Pradeep, T. Atomically precise clusters of noble metals: emerging link between atoms and nanoparticles. Chem. Rev. 2017, 117 (12), 8208-8271.

(3) Kurashige, W.; Niihori, Y.; Sharma, S.; Negishi, Y. Precise synthesis, functionalization and application of thiolate-protected gold clusters. Coord. Chem. Rev. 2016, 320-321, 238-250.

(4) Fuhr, O.; Dehnen, S.; Fenske, D. Chalcogenide clusters of copper and silver from silylated chalcogenide sources. Chem. Soc. Rev. 2013, 42 (4), 1871-1906.

(5) Jin, R.; Pei, Y.; Tsukuda, T. Controlling nanoparticles with atomic precision. Acc. Chem. Res. 2019, 52 (1), 1-1.

(6) Kalmutzki, M. J.; Hanikel, N.; Yaghi, O. M. Secondary building units as the turning point in the development of the reticular chemistry of MOFs. Sci. Adv. 2018, 4 (10), No. eaat9180.

(7) Guillerm, V.; Weseliński, Ł. J.; Belmabkhout, Y.; Cairns, A. J.; D'Elia, V.; Wojtas, Ł.; Adil, K.; Eddaoudi, M. Discovery and introduction of a $(3,18)$-connected net as an ideal blueprint for the design of metal-organic frameworks. Nat. Chem. 2014, 6, 673.

(8) Alezi, D.; Peedikakkal, A. M. P.; Weseliński, Ł. J.; Guillerm, V.; Belmabkhout, Y.; Cairns, A. J.; Chen, Z.; Wojtas, Ł.; Eddaoudi, M. Quest for highly connected metal-organic framework platforms: rareearth polynuclear clusters versatility meets net topology needs. J. Am. Chem. Soc. 2015, 137 (16), 5421-5430.

(9) Ji, Z.; Trickett, C.; Pei, X.; Yaghi, O. M. Linking Molybdenumsulfur clusters for electrocatalytic hydrogen evolution. J. Am. Chem. Soc. 2018, 140 (42), 13618-13622.

(10) Schoedel, A.; Yaghi, O. M. Reticular chemistry of metalorganic frameworks composed of copper and zinc metal oxide secondary building units as nodes. In The Chemistry of Metal-Organic Frameworks; Kaskel, S., Ed.; Wiley-VCH Verlag GmbH \& Co. KGaA, 2016; pp 43-72.

(11) Eddaoudi, M.; Moler, D. B.; Li, H.; Chen, B.; Reineke, T. M.; O'Keeffe, M.; Yaghi, O. M. Modular Chemistry: Secondary Building units as a basis for the design of highly porous and robust metalorganic carboxylate frameworks. Acc. Chem. Res. 2001, 34 (4), 319330.

(12) Yaghi, O. M.; O’Keeffe, M.; Ockwig, N. W.; Chae, H. K.; Eddaoudi, M.; Kim, J. Reticular synthesis and the design of new materials. Nature 2003, 423, 705.

(13) Joshi, C. P.; Bootharaju, M. S.; Bakr, O. M. Tuning properties in silver clusters. J. Phys. Chem. Lett. 2015, 6 (15), 3023-3035.

(14) Rais, D.; Yau, J.; Mingos, D. M. P.; Vilar, R.; White, A. J. P.; Williams, D. J. Anion-templated syntheses of rhombohedral silveralkynyl cage compounds. Angew. Chem., Int. Ed. 2001, 40 (18), 34643467.

(15) Liu, C.; Li, T.; Abroshan, H.; Li, Z.; Zhang, C.; Kim, H. J.; Li, G.; Jin, R. Chiral Ag23 nanocluster with open shell electronic structure and helical face-centered cubic framework. Nat. Commun. 2018, 9 (1), 744.

(16) Joshi, C. P.; Bootharaju, M. S.; Alhilaly, M. J.; Bakr, O. M. [Ag25(SR)18]-: the "golden" silver nanoparticle. J. Am. Chem. Soc. 2015, 137 (36), 11578-11581.

(17) AbdulHalim, L. G.; Bootharaju, M. S.; Tang, Q.; Del Gobbo, S.; AbdulHalim, R. G.; Eddaoudi, M.; Jiang, D.-E.; Bakr, O. M. Ag29(BDT)12(TPP)4: a tetravalent nanocluster. J. Am. Chem. Soc. 2015, 137 (37), 11970-11975.

(18) Desireddy, A.; Conn, B. E.; Guo, J.; Yoon, B.; Barnett, R. N.; Monahan, B. M.; Kirschbaum, K.; Griffith, W. P.; Whetten, R. L.;
Landman, U.; Bigioni, T. P. Ultrastable silver nanoparticles. Nature 2013, 501 (7467), 399-402.

(19) Yang, H.; Wang, Y.; Huang, H.; Gell, L.; Lehtovaara, L.; Malola, S.; Häkkinen, H.; Zheng, N. All-thiol-stabilized Ag44 and Au12Ag32 nanoparticles with single-crystal structures. Nat. Commun. 2013, 4, 2422.

(20) Jin, S.; Wang, S.; Song, Y.; Zhou, M.; Zhong, J.; Zhang, J.; Xia, A.; Pei, Y.; Chen, M.; Li, P.; Zhu, M. Crystal structure and optical properties of the [Ag62S12(SBut)32]2+ nanocluster with a complete face-centered cubic kernel. J. Am. Chem. Soc. 2014, 136 (44), 1555915565 .

(21) Wang, Z.; Su, H.-F.; Kurmoo, M.; Tung, C.-H.; Sun, D.; Zheng, L.-S. Trapping an octahedral Ag6 kernel in a seven-fold symmetric Ag56 nanowheel. Nat. Commun. 2018, 9 (1), 2094.

(22) Alhilaly, M. J.; Bootharaju, M. S.; Joshi, C. P.; Besong, T. M.; Emwas, A.-H.; Juarez-Mosqueda, R.; Kaappa, S.; Malola, S.; Adil, K.; Shkurenko, A.; Häkkinen, H.; Eddaoudi, M.; Bakr, O. M. [Ag67(SPhMe2)32(PPh3)8]3+: synthesis, total structure, and optical properties of a large box-shaped silver nanocluster. J. Am. Chem. Soc. 2016, 138 (44), 14727-14732.

(23) Chai, J.; Yang, S.; Lv, Y.; Chen, T.; Wang, S.; Yu, H.; Zhu, M. A unique pair: $\mathrm{Ag} 40$ and $\mathrm{Ag} 46$ nanoclusters with the same surface but different cores for structure-property correlation. J. Am. Chem. Soc. 2018, 140 (46), 15582-15585.

(24) Bodiuzzaman, M.; Ghosh, A.; Sugi, K. S.; Nag, A.; Khatun, E.; Varghese, B.; Paramasivam, G.; Antharjanam, S.; Natarajan, G.; Pradeep, T. Camouflaging structural diversity: co-crystallization of two different nanoparticles having different cores but the same shell. Angew. Chem., Int. Ed. 2019, 58 (1), 189-194.

(25) Song, Y.; Lambright, K.; Zhou, M.; Kirschbaum, K.; Xiang, J.; Xia, A.; Zhu, M.; Jin, R. Large-scale synthesis, crystal structure, and optical properties of the Ag146Br2(SR) 80 nanocluster. ACS Nano 2018, 12 (9), 9318-9325.

(26) Yang, H.; Wang, Y.; Chen, X.; Zhao, X.; Gu, L.; Huang, H.; Yan, J.; Xu, C.; Li, G.; Wu, J.; Edwards, A. J.; Dittrich, B.; Tang, Z.; Wang, D.; Lehtovaara, L.; Häkkinen, H.; Zheng, N. Plasmonic twinned silver nanoparticles with molecular precision. Nat. Commun. 2016, 7, 12809.

(27) Li, S.; Du, X.-S.; Li, B.; Wang, J.-Y.; Li, G.-P.; Gao, G.-G.; Zang, S.-Q. Atom-precise modification of silver(i) thiolate cluster by shell ligand substitution: a new approach to generation of cluster functionality and chirality. J. Am. Chem. Soc. 2018, 140 (2), 594-597.

(28) Zhao, S.; Jin, R.; Jin, R. Opportunities and challenges in $\mathrm{CO} 2$ Reduction by gold- and silver-based electrocatalysts: from bulk metals to nanoparticles and atomically precise nanoclusters. ACS Energy Lett. 2018, 3 (2), 452-462.

(29) Yan, J.; Zhang, J.; Chen, X.; Malola, S.; Zhou, B.; Selenius, E.; Zhang, X.; Yuan, P.; Deng, G.; Liu, K.; Su, H.; Teo, B. K.; Häkkinen, H.; Zheng, L.; Zheng, N. Thiol-stabilized atomically precise, superatomic silver nanoparticles for catalysing cycloisomerization of alkynyl amines. Natl. Sci. Rev. 2018, 5 (5), 694-702.

(30) Chang, Z.; Jing, X.; He, C.; Liu, X.; Duan, C. Silver clusters as robust nodes and $\pi$-activation sites for the construction of heterogeneous catalysts for the cycloaddition of propargylamines. ACS Catal. 2018, 8 (2), 1384-1391.

(31) Zhang, J.; Cheng, F.; Li, J.; Zhu, J.-J.; Lu, Y. Fluorescent nanoprobes for sensing and imaging of metal ions: recent advances and future perspectives. Nano Today 2016, 11 (3), 309-329.

(32) Song, X.-R.; Goswami, N.; Yang, H.-H.; Xie, J. Functionalization of metal nanoclusters for biomedical applications. Analyst 2016, 141 (11), 3126-3140.

(33) Zheng, K.; Setyawati, M. I.; Leong, D. T.; Xie, J. Antimicrobial silver nanomaterials. Coord. Chem. Rev. 2018, 357, 1-17.

(34) Huang, R.-W.; Wei, Y.-S.; Dong, X.-Y.; Wu, X.-H.; Du, C.-X.; Zang, S.-Q.; Mak, T. C. W. Hypersensitive dual-function luminescence switching of a silver-chalcogenolate cluster-based metalorganic framework. Nat. Chem. 2017, 9, 689.

(35) Wang, Z.-Y.; Wang, M.-Q.; Li, Y.-L.; Luo, P.; Jia, T.-T.; Huang, R.-W.; Zang, S.-Q.; Mak, T. C. W. Atomically precise site-specific 
tailoring and directional assembly of superatomic silver nanoclusters. J. Am. Chem. Soc. 2018, 140 (3), 1069-1076.

(36) Dong, X.-Y.; Huang, H.-L.; Wang, J.-Y.; Li, H.-Y.; Zang, S.-Q. A flexible fluorescent SCC-MOF for switchable molecule identification and temperature display. Chem. Mater. 2018, 30 (6), 2160-2167.

(37) Wang, Z.; Li, X.-Y.; Liu, L.-W.; Yu, S.-Q.; Feng, Z.-Y.; Tung, C.H.; Sun, D. Beyond clusters: supramolecular networks self-assembled from nanosized silver clusters and inorganic anions. Chem. - Eur. J. 2016, 22 (20), 6830-6836.

(38) Chen, Z.-Y.; Tam, D. Y. S.; Zhang, L. L.-M.; Mak, T. C. W. Silver thiolate nano-sized molecular clusters and their supramolecular covalent frameworks: an approach toward pre-templated synthesis. Chem. - Asian J. 2017, 12 (20), 2763-2769.

(39) Huang, R.-W.; Dong, X.-Y.; Yan, B.-J.; Du, X.-S.; Wei, D.-H.; Zang, S.-Q.; Mak, T. C. W. Tandem silver cluster isomerism and mixed linkers to modulate the photoluminescence of clusterassembled materials. Angew. Chem., Int. Ed. 2018, 57 (28), 85608566.

(40) Wang, Q.-M.; Lin, Y.-M.; Liu, K.-G. Role of anions associated with the formation and properties of silver clusters. Acc. Chem. Res. 2015, 48 (6), 1570-1579.

(41) Johnson, N. W. Convex polyhedra with regular faces. Can. J. Math. 1966, 18, 169-200.

(42) Bian, S.-D.; Wang, Q.-M. Snowman-like silver alkynyl cluster consolidated by templating chloride and peripheral trifluoroacetates. Chem. Commun. 2008, No. 43, 5586-5588.

(43) Zhang, S.-S.; Su, H.-F.; Zhuang, G.-L.; Wang, X.-P.; Tung, C.H.; Sun, D.; Zheng, L.-S. A hexadecanuclear silver alkynyl cluster based $\mathrm{NbO}$ framework with triple emissions from the visible to nearinfrared II region. Chem. Commun. 2018, 54 (84), 11905-11908.

(44) Spek, A. PLATON SQUEEZE: a tool for the calculation of the disordered solvent contribution to the calculated structure factors. Acta Crystallogr., Sect. C: Struct. Chem. 2015, 71 (1), 9-18.

(45) Sleigh, C.; Pijpers, A. P.; Jaspers, A.; Coussens, B.; Meier, R. J. On the determination of atomic charge via ESCA including application to organometallics. J. Electron Spectrosc. Relat. Phenom. 1996, 77 (1), 41-57.

(46) Ward, R. L. A complete introduction to modern NMR spectroscopy (Macomber, Rodger S.). J. Chem. Educ. 1999, 76 (4), 473.

(47) Li, G.; Lei, Z.; Wang, Q.-M. Luminescent molecular Ag-S nanocluster [Ag62S13(SBut)32](BF4)4. J. Am. Chem. Soc. 2010, 132 (50), 17678-17679. 